\title{
Fractional topological phase in one-dimensional flat bands with nontrivial topology
}

\author{
Huaiming Guo, ${ }^{1}$ Shun-Qing Shen, ${ }^{2}$ and Shiping Feng ${ }^{3}$ \\ ${ }^{1}$ Department of Physics, Beihang University, Beijing, 100191, China \\ ${ }^{2}$ Department of Physics, The University of Hong Kong, Pokfulam Road, Hong Kong \\ ${ }^{3}$ Department of Physics, Beijing Normal University, Beijing, 100875, China
}

(Received 11 April 2012; revised manuscript received 1 August 2012; published 17 August 2012)

\begin{abstract}
We show the existence of the fractional topological phase (FTP) in a one-dimensional interacting fermion model using exact diagonalization, in which the noninteracting part has flat bands with nontrivial topology. In the presence of the nearest-neighboring interaction $V_{1}$, the FTP at filling factor $v=1 / 3$ appears. It is characterized by the threefold degeneracy and the quantized total Berry phase of the ground states. The FTP is destroyed by a next-nearest-neighboring interaction $V_{2}$, and the phase diagrams in the $\left(V_{1}, V_{2}\right)$ plane are determined. We also present a physical picture of the phase and discuss its existence in the nearly flat band. Within the picture, we argue that the FTP at other filling factors can be generated by introducing proper interactions. The present study contributes to a systematic understanding of the FTPs and can be realized in cold-atom experiments.
\end{abstract}

DOI: 10.1103/PhysRevB.86.085124

PACS number(s): 71.10.Fd, 05.30.Fk, 73.43.Cd

\section{INTRODUCTION}

The discovery of integer (IQHE) and fractional (FQHE) quantum Hall effects opened a window to explore the mystery in condensed matter physics. ${ }^{1-3}$ They are topological states of quantum matter, which go beyond the Landau's theory of spontaneous symmetry breaking. ${ }^{4}$ The studies of these effects enrich our understanding of quantum phases and quantum phase transitions. In his seminal paper, ${ }^{5}$ Haldane showed that IQHE can be realized on a lattice model without a net magnetic field. The most recent generalization of the Haldane model to electrons with spin $1 / 2$ gives birth to the time-reversal invariant $Z_{2}$ topological insulator (TI), which becomes the current research focus in condensed matter physics due to their many exotic properties. ${ }^{6-10}$

Inspired by this, similar ideas arise for the situation of FQHE, and many studies are devoted to realizing the fractional topological phase (FTP) on lattice models in the absence of external magnetic fields. Up to now great development has been achieved. Models that exhibit a nearly flat band with a nonzero Chern number are proposed in different systems, and numerical calculations confirm its existence when interactions are included. ${ }^{11-20}$ To gain some insight of FTP, in this paper we focus on the one-dimensional (1D) case. Using exact diagonalization, we calculate the low-energy spectrum and Berry phase of the lowest energy states of a 1D interacting topological flat-band model, and identify the FTP at the filling factor, i.e., the average number of electrons per site, $v=1 / 3$. We also present a physical picture of the phase and discuss the possible existence of fractional charges in the nearly flat band. The FTPs at other filling factors can be generated by introducing proper interactions.

\section{1D TOPOLOGICAL FLAT-BAND MODEL}

Consider the 1D noninteracting tight-binding model, ${ }^{21}$

$$
\begin{aligned}
H_{0}= & \sum_{i}(M+2 B) \Psi_{i}^{\dagger} \sigma_{z} \Psi_{i}-\sum_{i, \hat{x}} B \Psi_{i}^{\dagger} \sigma_{z} \Psi_{i+\hat{x}} \\
& -\sum_{i, \hat{x}} \operatorname{sgn}(\hat{x}) i A \Psi_{i}^{\dagger} \sigma_{x} \Psi_{i+\hat{x}},
\end{aligned}
$$

where $\sigma_{x}$ and $\sigma_{z}$ are Pauli matrices, and the spinor $\Psi_{i}=$ $\left(c_{i}, d_{i}\right)^{T}$ with $c_{i}\left(d_{i}\right)$ electron annihilating operator at the site $\mathbf{r}_{i}$, which can be obtained by mapping the Dirac equation into a lattice. ${ }^{22}$ In the momentum space Eq. (1) becomes $H_{0}=\sum_{k} \Psi_{k}^{\dagger} \mathcal{H}(k) \Psi_{k}$ with $\Psi_{k}=\left(c_{k}, d_{k}\right)^{T}$, the Fourier partner of $\Psi_{i}$ and

$$
\mathcal{H}(k)=[M+2 B-2 B \cos (k)] \sigma_{z}+2 A \sin (k) \sigma_{x} .
$$

The spectrum of $\mathcal{H}(k)$ consists of two bands,

$$
E_{k}^{(1,2)}= \pm \sqrt{[M+2 B-2 B \cos (k)]^{2}+[2 A \sin (k)]^{2}} .
$$

Usually the two bands are dispersive, but when the parameters satisfy $-M / 2=B=A$, the bands are flat, when $E_{k}= \pm|2 A|$. Also the resulting flat band has a nontrivial Berry phase $\pi$ $\bmod (2 \pi)$, which is manifested by the existence of the zero energy modes at the two ends of the system. Thus we realize the topological flat band in one dimension.

\section{THE EFFECT OF INTERACTION}

Next we study the effect of interactions in the topological flat band. We firstly add nearest-neighboring (NN) and nextnearest-neighboring (NNN) interactions to Eq. (1), which are written as

$$
H_{I}=V_{1} \sum_{\langle i, j\rangle} n_{i} n_{j}+V_{2} \sum_{\langle\langle i, j\rangle\rangle} n_{i} n_{j},
$$

where $n_{i}=c_{i}^{\dagger} c_{i}+d_{i}^{\dagger} d_{i}$ is the total number of electrons on site $\mathbf{r}_{i}$ and $V_{1}, V_{2}$ are the strength of the interactions. We perform the exact diagonalization study of the total Hamiltonian $H_{0}+H_{I}$ on a finite chain of $N$ sites with a periodic boundary condition. We denote the number of particles as $N_{p}$, and the filling factor of the topological flat band is $v=N_{p} / N$. We have carried out the calculations at $v=1 / 3$ and identified the FTP in which the ground states are threefold degenerate. We firstly glance at the phase diagram in the $\left(V_{1}, V_{2}\right)$ plane, which is shown is Fig. 1(c). By turning on $V_{1}$, the ground state is threefold degenerate and the FTP emerges. The ground state is separated from higher eigenstates by a finite gap $\Delta$. As shown in Fig. 2(a), the value of the gap increases with the 

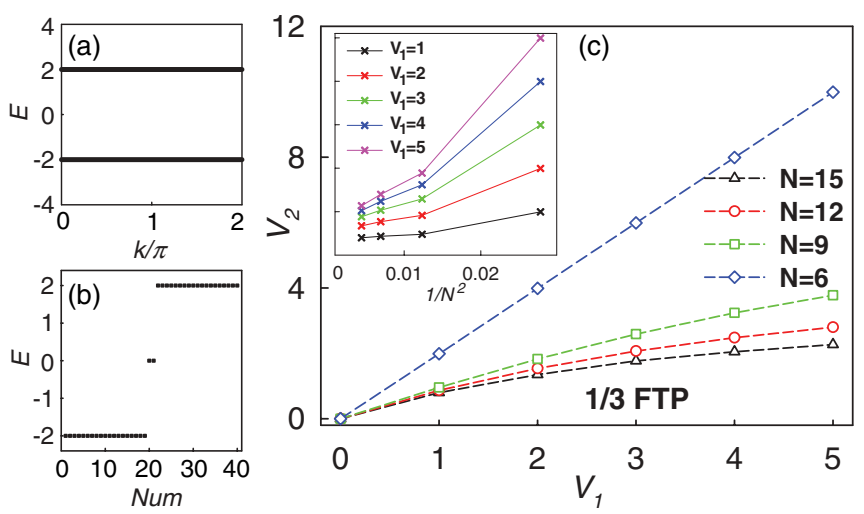

FIG. 1. (Color online) (a) The tight-binding band structure when the parameters satisfy $-M / 2=B=A$, where the bands are flat. (b) Edge modes in the flat-band case on a chain of length $N=20$ with open boundary condition. (c) The phase diagram in the $\left(V_{1}, V_{2}\right)$ plane at $v=1 / 3$ for different sizes. The inset shows the size dependence of the critical value $V_{2 c}$ at different $V_{1}$. Here $A=B=1$ and $M=-2$ (in the following calculations if not mentioned, we use these parameters).

strength of $V_{1}$, indicating that the FTP is more stable at larger $V_{1}$. After turning on $V_{2}$, the value of the gap vanishes and the FTP is destroyed at a critical value $V_{2 c}$ [see Fig. 2(b)], which marks the boundary of the FTP in the phase diagram. We determine the boundaries of the FTP on different sizes of the chain and find that the region of the FTP is shrunk as the size increases. However according to the results on the sizes we can access, it is reasonable to deduce that the FTP exists in the thermodynamic limit.

The system under consideration has translational symmetry, and the momentum of the eigenstate is a good quantum number. Thus the Hamiltonian can be diagonalized in each sector with the momentum $q=2 \pi k / N(k=0,1, \ldots, N-1)$, which allows us to examine the character of the low-energy spectrum in the momentum space. In Fig. 2(c) we show the ground-state energy of each momentum sector at $V_{1}=1$ and
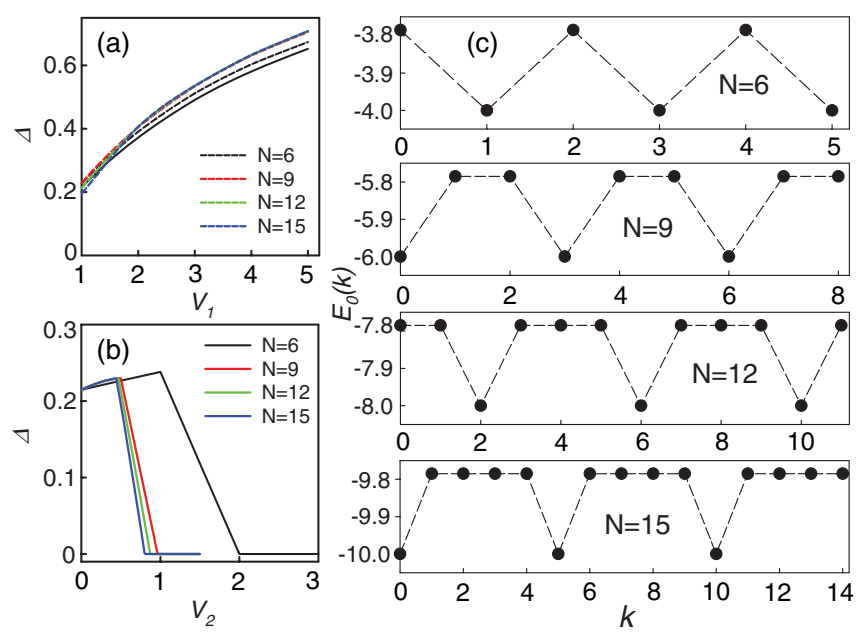

FIG. 2. (Color online) (a) The gap between the ground states and the excited states versus $V_{1}$ at $V_{2}=0$ (solid line) and $V_{2}=0.5$ (dashed line). (b) The gap versus $V_{2}$ at $V_{1}=1$. (c) The ground-state energy of each momentum sector at $V_{1}=1$ and $V_{2}=0$ on systems with different sizes.
$V_{2}=0$ on systems with different sizes. It has been shown that for these parameters the ground states are threefold degenerate. Here we further demonstrate that the three states are in different momentum sectors. If $k_{1}$ is the momentum sector for one of the ground-state manifold, the other states should be obtained in the sectors $k_{1}+N_{p}$ and $k_{1}+2 N_{p}$ (module $N$ ). For the cases with nonzero $V_{2}$ the results are similar. The gap between the ground states and the excited states is clearly demonstrated in Fig. 2(c). We find that the value of the gap is independent of the size of the system for $V_{2}=0$ [the solid line in Fig. 2(a)], while for $V_{2} \neq 0$ it is dependent [the dashed line in Fig. 2(a)].

To further confirm the existence of the FTP, we study the topological property of the ground states. It can be understood in terms of the total Berry phase of the ground states. By using the twisted boundary conditions, it is expressed as ${ }^{23,24}$

$$
\gamma=\sum_{j=1}^{3} \oint i\left\langle\psi_{\theta}^{j}\left|\frac{d}{d \theta}\right| \psi_{\theta}^{j}\right\rangle,
$$

where $\theta$ is the twisted boundary phase which takes values from 0 to $2 \pi$ and $\psi_{\theta}^{j}$ are the corresponding many-body wave functions of the threefold degenerate ground states. Since the flat band has nontrivial topology, the total Berry phase of the ground states is expected to be $\bmod (\gamma, 2 \pi)=\pi$. Each ground state shares the Berry phase $\pi / 3$ averagely. When the bands are exactly flat, the energy of the ground states remains the same as $\theta$ varies. So to perform the calculations, we adjust the parameter $M$ a little and make the band nearly flat. As shown in Fig. 3(a), the ground-state energies are slightly split and vary with $\theta$. Then we calculate the total Berry phase at different $V_{1}$ on a chain with $N=6$ and show the result in Fig. 3(c). It shows that the total Berry phase gets a nontrivial value $\pi$ for small $V_{2}$ and jumps to zero as $V_{2}$ is further increased. The obtained critical values $V_{2 c}$ are consistent with those from the energy spectra. Moreover, for other lattice sizes the
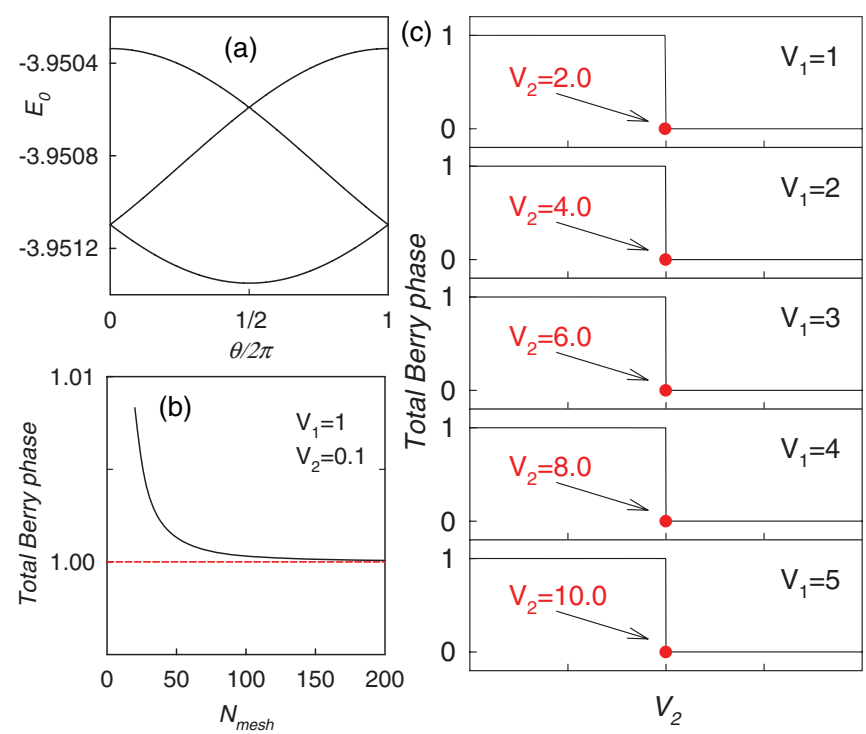

FIG. 3. (Color online) (a) The energies of the ground states versus $\theta$ at $V_{1}=1$ and $V_{2}=0$. (b) The total Berry phase versus the number of divided meshes $N_{\text {mesh }}$. (c) The total Berry phase versus $V_{2}$ at different fixed $V_{1}$. Here the parameters are $A=B=1$ and $M=-1.99$ when the band slightly departs exact flatness. 
results are the same. In the calculations, we divide the range of the boundary phase $[0,2 \pi]$ into $N_{\text {mesh }}=100$ meshes, which allows the results to have acceptable precision. With the above methods, we also identify the FTP at $v=1 / 4$.

\section{MAPPING TO THE SU-SCHRIEFFER-HEEGER MODEL}

The Hamiltonian described by Eq. (1) can be obtained through a dimensional reduction from the Bernevig-HughesZhang model which describes the two-dimensional topological insulator HgTe. ${ }^{25}$ Performing a cyclic permutation of the Pauli matrices $\sigma_{z} \rightarrow \sigma_{x}, \sigma_{x} \rightarrow \sigma_{y}$ and $\sigma_{y} \rightarrow \sigma_{z}$ in Eq. (1), the Hamiltonian becomes

$$
\begin{aligned}
H_{0}^{\prime}= & \left.\sum_{i}(M+2 B)\left[c_{i}^{\dagger} d_{i}+d_{i}^{\dagger} c_{i}\right)\right]-\sum_{i, \hat{x}}\left([B+\operatorname{sgn}(\hat{x}) A] c_{i}^{\dagger} d_{i+\hat{x}}\right. \\
& \left.+[B-\operatorname{sgn}(\hat{x}) A] d_{i}^{\dagger} c_{i+\hat{x}}\right),
\end{aligned}
$$

where the hopping amplitudes are all real. If the two orbits on each site are viewed as two separate sites, it describes the free electrons on a chain with NN and next-next-nearestneighboring hoppings, as shown in Fig. 4(a). In the flat-band case of $A=B=-M / 2$, it can be simplified as

$$
H_{0, \text { flat }}^{\prime}=-2 A\left(\sum_{i} c_{i}^{\dagger} d_{i+1}+d_{i+1}^{\dagger} c_{i}\right),
$$

which is the Su-Schrieffer-Heeger model in the limit case where only NN hopping exists and every other bond is broken completely. ${ }^{26,27}$ The model is solvable by setting $\gamma_{i, \pm}=\left(c_{i} \pm\right.$ $\left.d_{i+1}\right) / \sqrt{2}$. Then $H_{0, \text { flat }}^{\prime}=-2 A\left(\sum_{i} \gamma_{i,+}^{\dagger} \gamma_{i,+}-\gamma_{i,-}^{\dagger} \gamma_{i,-}\right)$, and then we have the two flat-band spectra as shown in Fig. 1(a). At $v=1 / 3$, the lowest energy state is highly degenerate. In the presence of the $\mathrm{NN}$ interaction in Eq. (1), there are three configurations of the ground states where the NN interaction between the electrons can be minimized, $\left|g_{, \alpha}\right\rangle=\Pi_{n} \gamma_{3 n+\alpha,+}^{\dagger}|0\rangle$ with $\alpha=0,1,2$. These three states $\left|g_{, \alpha}\right\rangle$ are the charge-density wave as depicted in Fig. 4(b), which breaks the translational symmetry. However, we found that the lowest energy state in each momentum sector $K$ has a uniform density distribution of electron, which is consistent with the translational symmetry

(a)

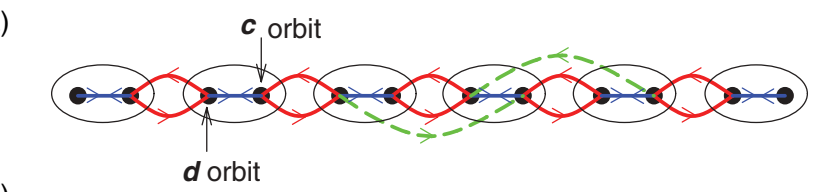

(b)

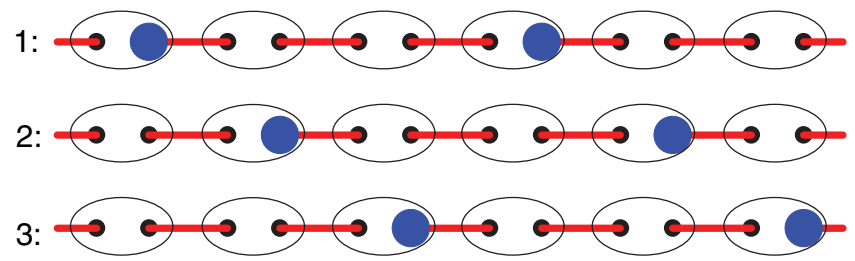

FIG. 4. (Color online) (a) The schematic representation of the resulting Hamiltonian after rotating the Pauli matrices. The hopping amplitudes of the blue, red, and green bonds are $M+2 B,-(B+A)$, and $-(B-A)$ respectively. (b) The three degenerate ground states at $v=1 / 3$. in Eq. (1). The states are the linear combination of the three degenerate states $\left|g_{, \alpha}\right\rangle$. Utilizing the lattice translational operator, we can construct the three states as eigenstates of the momentum $K:|g, K\rangle=\frac{1}{\sqrt{3}} \sum_{\alpha} e^{i \alpha K}\left|g_{, \alpha}\right\rangle$ where $K=2 n \pi / 3$ $(n=0,1,2)$. Thus these three states are those in the presence of interactions. It can be checked as a limit of the nearly flat band, in which the density distribution of electrons is always uniform. Therefore the ground states show threefold degeneracy. After the NNN interaction is turned on though, the electrons begin to interact with each other, the three configurations still have the same lowest energies for small $V_{2}$, and the threefold degenerate ground states persist for $V_{2}<V_{2 c}$. In particular with the above picture $n$-fold ( $n$ other than three or four) degenerate ground states can be generated by introducing proper interactions.

\section{THE FTP IN THE NEARLY FLAT BAND}

Up to now we have established the existence of the FTP in the topological flat band. It is natural to ask whether the phase persists in the nearly flat band. In the following we let the band disperse by tuning the parameter $M$ and study the properties of the ground states. As shown in Fig. 5(a), when $\delta M=2-M \neq 0$ the energy of the three lowest energy states are split. An energy gap $\Delta_{1}$ appears between the lower twofold degenerate states and the upper one, whose value increases with $\delta M$. Meanwhile the energy gap $\Delta_{2}$ separating the three lowest energy states from the fourth lowest energy state decreases with $\delta M$ and vanishes at a critical value $\delta M_{c}$. Moreover beyond $\delta M_{c}$ the total Berry phase of the three states with the lowest energies is no longer quantized to $\pi$, which implies the occurrence of a topological quantum phase transition. Now the question becomes whether the three lowest states are still degenerate for $0<\delta M<\delta M_{c}$ in the thermodynamic limit. To this end, the finite-size analysis of two energy gaps $\Delta_{1}$ and $\Delta_{2}$ at several small $\delta M$ is presented in Figs. 5(b) and 5(c). The results demonstrate that $\Delta_{1}$ tends to vanish while $\Delta_{2}$ survives in the thermodynamic limit, i.e., for a large $N$ limit. So the splitting of the degeneracy of the
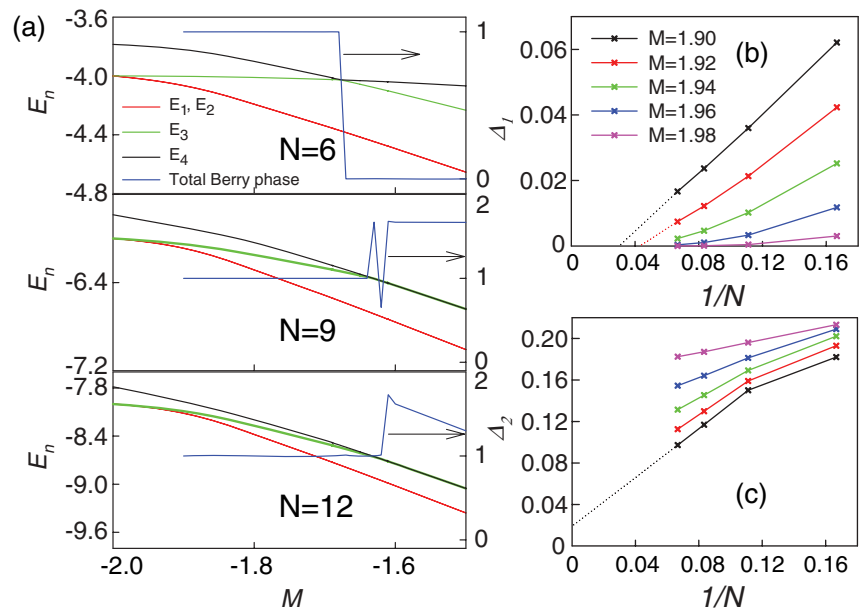

FIG. 5. (Color online) (a) The energies of the lowest four states and the total Berry phase of the lowest three states versus $M$. (b) and (c) The finite-size analysis of $\Delta_{1}$ and $\Delta_{2}$, respectively. Here $v=1 / 3$. 
lowest energy states may be due to the finite-size effect, and the FTP persists in the nearly flat band.

\section{CONCLUSIONS}

We have studied the FTP in a 1D interacting topological flat-band model using exact diagonalization. By introducing the NN and NNN interactions, we identify the FTP at $v=1 / 3$, in which the ground states are threefold degenerate. Moreover the degenerate ground states are in different momentum sectors and are equally spaced with the interval of $N_{p}$. With the above method, we have obtained the phase diagram at $v=1 / 3$ in the $\left(V_{1}, V_{2}\right)$ plane. We also study the total Berry phase of the low-energy states and find that it gets a quantized value $\pi$ when the system is in the FTP. The phase boundary from the total Berry phase is consistent with that from the low-energy spectrum.

The existence of the 1D FTP is closely related to the underlying physics in dimerized polyacetylene. ${ }^{25-27}$ One explicit consequence of the 1D FTP is the charge fractionalization of the quasiparticles or excitations. Following Kivelson and Schrieffer, ${ }^{28}$ in the threefold degenerate ground states, the domain walls or solitons possess the fractional charges of $e / 3$, which can be changed by an integer by adding electrons or holes. In our case, suppose a domain wall is formed between two of the three degenerate ground states. The domain wall can move freely on the chain. When we move the particle one unit, the domain wall moves three units. Since the change in electric dipole moment can be calculated in two equivalent ways, i.e., through the particle motion $e(+1)$ and through the domain wall motion $Q(+3)$, we have $Q=e / 3$, which is the fractional excitation associated with the domain wall of the $1 \mathrm{D}$ FTP. For strongly interacting systems, it can be also understood very well from the bosonization and the theory of macroscopic polarization by calculating the Berry phases. ${ }^{29}$

Now we can have a systematic understanding of the FTPs on lattices without net magnetic field in all three dimensions. For the 1D case, the flat band has a nontrivial Berry phase and the excitations with fractional charges characterize the FTP. For the 2D case, the FTP is found in interacting electrons of the flat band with a nontrivial Chern number. Its feature is almost multifold degenerate incompressible ground states with fractional Hall conductance, which is similar to FQHE.
Also by combining the two decoupled FTPs formed by spin up and down electrons, we have fractional quantum spin Hall effect or fractional TI with time-reversal symmetry. For the 3D case in the nearly flat band characterized by a nontrivial $Z_{2}$ topological index and in the presence of repulsive interactions, a 3D fractional TI can be generated. Thus FTP can be realized in interacting electrons with topologically nontrivial bands. The common features are the multifold degeneracy of the ground states and topological invariants of all degenerated ground states.

Due to the rapid development of the field of cold atoms, ${ }^{30}$ it is very hopeful to realize the $1 \mathrm{D}$ FTP on state-dependent optical lattice with laser-assisted tunneling between adjacent sites. ${ }^{31-33}$ To simulate our Hamiltonian, atoms with four internal states $c_{1,2}$ and $d_{1,2}$ are required, such as ${ }^{6} \mathrm{Li}$. Then in the state-dependent optical lattice, atoms in states $c_{1}$ and $d_{1}$ are at positions $\mathbf{r}_{1}=2 n$ and atoms in states $c_{2}$ and $d_{2}$ are at positions $\mathbf{r}_{2}=2 n+1$, where the lattice constant is $\lambda / 4$ with $\lambda$ the wavelength of the laser generating the optical lattice. We can choose the optical potential large enough to prohibit direct tunneling between neighboring sites. The hoppings in the model are induced by additional lasers driving Raman transitions between different states. By setting different resonance frequencies for different kinds of hopping, the noninteracting Hamiltonian can be realized using proper additional lasers. Since the separation between neighboring sites is half the original lattice constant, significant NN interactions can be generated. Also due to the fact that on-site interactions don't affect the FTP, it is very possible to realize the FTP in this scheme. Finally it would be exciting to find real materials exhibiting the FTP.

\section{ACKNOWLEDGMENTS}

The authors would like to thank Z.-C. Gu, Hua Jiang, D. N. Sheng, Jun-Ren Shi, and Yi-Fei Wang for helpful discussions. H.G. is supported by FOK YING TUNG EDUCATION FOUNDATION and NSFC under Grant No. 11104189; S.S. is supported by the Research Grant Council of Hong Kong under Grant No. N_HKU748/10; S.F. is supported by the Ministry of Science and Technology of China under Grant Nos. 2011CB921700 and 2012CB821403 and NSFC under Grant No. 11074023.

\footnotetext{
${ }^{1}$ K. V. Klitzing, G. Dorda, and M. Pepper, Phys. Rev. Lett. 45, 494 (1980).

${ }^{2}$ D. C. Tsui, H. L. Stormer, and A. C. Gossard, Phys. Rev. Lett. 48, 1559 (1982).

${ }^{3}$ R. B. Laughlin, Phys. Rev. Lett. 50, 1395 (1983).

${ }^{4}$ L. D. Landau and E. M. Lifschitz, Statistical Physics (Pergamon, London, 1958).

${ }^{5}$ F. D. M. Haldane, Phys. Rev. Lett. 61, 2015 (1988).

${ }^{6}$ C. L. Kane and E. J. Mele, Phys. Rev. Lett. 95, 226801 (2005).

${ }^{7}$ C. L. Kane and E. J. Mele, Phys. Rev. Lett. 95, 146802 (2005).

${ }^{8}$ J. E. Moore, Nature 464, 194 (2010).

${ }^{9}$ M. Z. Hasan and C. L. Kane, Rev. Mod. Phys. 82, 3045 (2010).
}

${ }^{10}$ X. L. Qi and S. C. Zhang, Rev. Mod. Phys. 83, 1057 (2011).

${ }^{11}$ E. Tang, J. W. Mei, and X. G. Wen, Phys. Rev. Lett. 106, 236802 (2011).

${ }^{12}$ T. Neupert, L. Santos, C. Chamon, and C. Mudry, Phys. Rev. Lett. 106, 236804 (2011).

${ }^{13}$ K. Sun, Z. Gu, H. Katsura, and S. Das Sarma, Phys. Rev. Lett. 106, 236803 (2011)

${ }^{14}$ F. Wang and Y. Ran, Phys. Rev. B 84, 241103 (2011).

${ }^{15}$ X. Hu, M. Kargarian, and G. A. Fiete, Phys. Rev. B 84, 155116 (2011).

${ }^{16}$ T. Neupert, L. Santos, S. Ryu, C. Chamon, and C. Mudry, Phys. Rev. B 84, 165107 (2011). 
${ }^{17}$ C. Weeks and M. Franz, Phys. Rev. B 85, 041104 (2012).

${ }^{18}$ X. L. Qi, Phys. Rev. Lett. 107, 126803 (2011).

${ }^{19}$ D. N. Sheng, Z. C. Gu, Kai Sun, and L. Sheng, Nature Communications 2, 389 (2011).

${ }^{20}$ Y. F. Wang, Z. C. Gu, C. D. Gong, and D. N. Sheng, Phys. Rev. Lett. 107, 146803 (2011).

${ }^{21}$ H.-M. Guo and S. Q. Shen, Phys. Rev. B 84, 195107 (2011).

${ }^{22}$ S. Q. Shen, W. Y. Shan, and H. Z. Lu, SPIN 1, 33 (2011).

${ }^{23}$ R. Resta, Rev. Mod. Phys. 66, 899 (1994).

${ }^{24}$ Q. Niu, D. J. Thouless, and Y.-S. Wu, Phys. Rev. B 31, 3372 (1985).

${ }^{25}$ B. A. Bernevig, T. L. Hughes, and S. C. Zhang, Science 314, 1757 (2006).
${ }^{26}$ W. P. Su, J. R. Schrieffer, and A. J. Heeger, Phys. Rev. Lett. 42, 1698 (1979).

${ }^{27}$ A. J. Heeger, S. Kivelson, J. R. Schrieffer, and W.-P. Su, Rev. Mod. Phys. 60, 781 (1988).

${ }^{28}$ S. Kivelson and J. R. Schrieffer, Phys. Rev. B 25, 6447 (1982).

${ }^{29}$ A. A. Aligia and C. D. Batista, Phys. Rev. B 71, 125110 (2005).

${ }^{30}$ I. Bloch, J. Dalibard, and W. Zwerger, Rev. Mod. Phys. 80, 885 (2008).

${ }^{31}$ N. Goldman, D. F. Urban, and D. Bercioux, Phys. Rev. A 83, 063601 (2011).

${ }^{32}$ D. Jaksch and P. Zoller, New J. Phys. 5, 56 (2003).

${ }^{33}$ F. Gerbier and J. Dalibard, New J. Phys. 12, 033007 (2010). 